\title{
AGN feedback in numerical simulations
}

\section{Luca Ciotti}

Department of Astronomy, University of Bologna, via Ranzani 1, 40127, Bologna, Italy email: luca.ciotti@unibo.it

\begin{abstract}
The passively evolving stellar population in elliptical galaxies (Es) provides a continuous source of fuel for accretion on the central supermassive black hole (SMBH), which is 1) extended over the entire galaxy life (but declining with cosmic time), 2) linearly proportional to the stellar mass of the host spheroid, 3) summing up to a total gas mass that is $>100$ times larger than the currently observed SMBH masses, 4) available independently of merging events. The main results of numerical simulations of Es with central SMBH, in which a physically based implementation of radiative and mechanical feedback effects is considered, are presented.
\end{abstract}

Keywords. X-rays: ISM - Galaxies: cooling flows - Galaxies: active

In a series of papers (Ciotti \& Ostriker 2007; Ciotti, Ostriker \& Proga 2009; Pellegrini, Ciotti \& Ostriker 2009; Shin, Ciotti \& Ostriker 2009; Jiang et al. 2009; see also Ciotti 2009) we study, with a high-resolution 1-D hydrodynamical code, the evolution of the ISM in Es under the action of SNIa heating, thermalization of the stellar mass losses, and feedback from the central SMBH. The cooling and heating functions include photoionization and Compton effects, radiation pressure is evaluated by solving the transport equation, mechanical feedback is produced by a physically based luminosity-dependent nuclear wind and jet, and star formation is also allowed. The recycled gas from the aging stars of the galaxy cools and collapses towards the center, a star-burst occurs and the central SMBH is fed. The energy output from the central SMBH pushes matter out, the accretion rate drops precipitously and the expanding matter drives shocks into the ISM. Then the resulting hot bubble ultimately cools and the consequent infall leads to renewed accretion; the cycle repeats, with the galaxy being seen alternately as an AGN/starburst for a small fraction of the time and as a "normal" elliptical hosting an incipient cooling catastrophe for much longer intervals. No steady flow appears to be possible for Eddington ratios above $\simeq 0.01$ : whenever the luminosity is significantly above this limit both the accretion and the output luminosity are in burst mode. Strong intermittencies are expected at early times, while at low redshift the models are characterized by smooth, very sub-Eddington mass accretion rates punctuated by rare outbursts. One of the general consequences of our exploration is the fact that the recycled gas from dying stars can induce substantial QSO activity, even in the absence of external phenomena such as galaxy merging, while accretion feedback can be strong enough to solve the "cooling-flow" problem and to maintain the mass of the SMBH on the observed range of values.

\section{References}

Ciotti, L. 2009, La Rivista del Nuovo Cimento 32, n.1, 1

Ciotti, L. \& Ostriker, J. P. 2007, ApJ 665, 1038

Ciotti, L., Ostriker, J. P., \& Proga, D. 2009, ApJ 699, 89

Pellegrini, S., Ciotti, L., \& Ostriker, J. P. 2009, Advances in Space Research 44, 340

Shin, M.-S., Ostriker, J. P., \& Ciotti, L. 2009, arXiv0905.4294

Jiang, Y. F., Ciotti, L., Ostriker, J. P., \& Spitkovsky, A. 2009, arXiv0904.4918 